\title{
El colesterol HDL: ¿un nuevo objetivo terapéutico en el manejo de las dislipidemias y la ateroesclerosis?
}

\author{
María del Rosario Laris $\mathrm{E}^{1}$, Antonio Arteaga $\mathrm{L}^{2}$, \\ Ada Cuevas $\mathbf{M}^{3}$, Attilio Rigotti $\mathbf{R}^{1}$. \\ HDL cholesterol: \\ A new target in the treatment of lipid \\ disorders and atherosclerosis?
}

Low plasma HDL cholesterol levels are an independent risk factor for atherosclerotic cardiovascular disease. During the past years, the study of HDL has been stimulated by the discovery of novel genes and proteins, which have provided new insights into the molecular and cellular mechanisms involved in HDL metabolism. In addition, recent clinical studies focused on treating low HDL as primary objective in high cardiovascular risk patients have been very encouraging. Furthermore, new drugs for raising HDL cholesterol are under development. This article reviews the recent progress in the HDL field and its important implications for the pathophysiology and treatment of atherosclerosis. In the near future, we expect that new drugs with specific and beneficial effects on HDL metabolism and the associated cardiovascular risk should improve the current approach to this disease with high burden on health systems and society (Rev Méd Chile 2005; 133: 823-32).

(Key Words: Atherosclerosis; Cardiovascular diseases; Lipoproteins, HDL cholesterol)

Recibido el 25 de agosto, 2004. Aceptado el 14 de diciembre, 2004.

Departamentos de ${ }^{1}$ Gastroenterología y ${ }^{2}$ Diabetes, Nutrición y Metabolismo, Facultad de Medicina, Pontificia Universidad Católica de Chile. ${ }^{3}$ Departamento de Medicina, Clínica Las Condes. Santiago, Chile.

$\mathrm{E}^{1}$ impacto de la enfermedad cardiovascular ateroesclerótica es especialmente relevante en regiones en transición epidemiológica como nuestro país ${ }^{1}$. Dentro de los factores de riesgo asociados, las dislipidemias constituyen uno de los

Correspondencia a: Dr. Attilio Rigotti. Facultad de Medicina, Pontificia Universidad Católica de Chile. Marcoleta 367, Santiago, Chile. E mail: arigotti@med.puc.cl elementos etiopatogénicos más significativos. Los niveles plasmáticos elevados de colesterol transportado en las lipoproteínas de baja densidad (CLDL) son un factor de riesgo pro-aterogénico ampliamente demostrado. Por otro lado, el beneficio de las estatinas, las cuales disminuyen el cLDL, en la prevención primaria y secundaria de la enfermedad cardiovascular está categóricamente establecido ${ }^{2}$. A pesar de la utilidad de las estatinas, todavía no se logra prevenir el 60 a $70 \%$ de 
los eventos cardiovasculares a 5-6 años plazo en los pacientes que reciben estos fármacos, lo que ha estimulado el estudio de nuevos blancos terapéuticos para el manejo de la ateroesclerosis.

Uno de estos nuevos blancos terapéuticos son los niveles plasmáticos del colesterol transportado en las lipoproteínas de alta densidad HDL (cHDL). Tanto estudios experimentales, epidemiológicos, genéticos y clínicos, indican que los niveles de cHDL se correlacionan en forma independiente e inversa con la presencia de enfermedad cardiovascular $^{3,4}$, indicando que las HDL constituyen un factor protector antiaterogénico. Por otro lado, niveles reducidos de cHDL constituyen la anormalidad lipídica más prevalente en sujetos con enfermedad coronaria precoz ${ }^{5}$. Además, estudios en modelos animales han demostrado la acción protectora vascular de las HDL. Este efecto antiaterogénico de las HDL involucraría múltiples mecanismos, incluyendo el transporte reverso del colesterol (ver más abajo) y una protección cardiovascular no relacionada directamente con la homeostasis del colesterol corporal ${ }^{6}$ (Tabla 1).

Recientemente, el campo de estudio del cHDL se ha revitalizado con el descubrimiento de nuevos genes y vías que participan en el metabolismo de las HDL. Además, el análisis retrospectivo de estudios clínicos previos y protocolos recientes enfocados hacia el manejo de los niveles de cHDL como objetivo terapéutico primario, han arrojado resultados promisorios. Esto ha llevado a considerar activamente el papel de los niveles de
cHDL en las estrategias de manejo de la enfermedad cardiovascular ateroesclerótica. Estos avances, que constituyen el tema central de esta revisión, tienen importantes implicancias para el diseño y la aplicación de nuevas medidas preventivas y terapéuticas de la ateroesclerosis.

\section{AVANCES RECIENTES EN EL ESTUDIO DEL METABOLISMO DE HDL}

Las HDL se sintetizan y secretan desde el hígado y el intestino como partículas nacientes de pre- $\Omega$ HDL o HDL discoidales, formadas predominantemente por apolipoproteína (apo) A-I y fosfolípidos. Estas partículas nacientes atraviesan el endotelio vascular de los tejidos periféricos, desde los cuales remueven el exceso de colesterol libre celular por acción del transportador de membrana $\mathrm{ABCA1}^{7}$ (Figura 1). ABCA1 se descubrió estudiando la enfermedad de Tangier, una condición patológica caracterizada por niveles muy reducidos de cHDL y causada por mutaciones en el gen que codifica este transportador ${ }^{7}$. La deficiencia de ABCA1 en los pacientes con enfermedad de Tangier origina un defecto en el eflujo celular de colesterol y fosfolípidos, lo que impide la maduración de las HDL nacientes, las cuales sufren un catabolismo acelerado ${ }^{7}$. La ausencia de expresión de ABCA1 en los macrófagos causa acumulación intracelular de colesterol y facilita la progresión de la ateroesclerosis ${ }^{8}$, lo que explicaría el riesgo

Tabla 1. Propiedades antiaterogénicas de las H D L

Mecanismos relacionados con el metabolismo lipídico

- Transporte reverso de colesterol y ésteres de colesterol oxidados

- Inhibición de la oxidación y la agregación de las LDL

- Prevención del daño vascular causado por las LDL oxidadas

Mecanismos no relacionados con el metabolismo lipídico

- Inhibición de la adherencia de los monocitos al endotelio y su migración a la íntima arterial

- Estimulación de la reparación endotelial

- Preservación de la reactividad vascular dependiente del endotelio

- Disminución del crecimiento y la proliferación de las células musculares lisas

- Prevención de la trombosis vascular 
cardiovascular elevado que se ha descrito en algunos pacientes con enfermedad de Tangier.

El colesterol libre captado por las HDL es esterificado con ácidos grasos por la enzima plasmática LCAT (lecithin cholesterol acyltransferase) formando ésteres de colesterol, que son movilizados hacia el interior de la partícula, determinando la formación de HDL esféricas maduras $^{9}$ (Figura 1). El cHDL puede ser removido de la circulación a través de diferentes procesos metabólicos (Figura 1). En mamíferos superiores, un primer mecanismo involucra la transferencia de los ésteres de colesterol desde las HDL hacia las lipoproteínas no HDL (VLDL y LDL) por acción de la enzima de transferencia de ésteres de colesterol (cholesteryl ester transfer protein, CETP). Este proceso determina que una fracción importante del colesterol transportado en las HDL sea metabolizado por medio de los receptores de la familia del receptor de LDL ${ }^{10}$. Por esta función, la CETP es un determinante clave de los niveles de cHDL. De hecho, hemos demostrado recientemente que un subgrupo de individuos chilenos con cHDL elevado presentaron una actividad

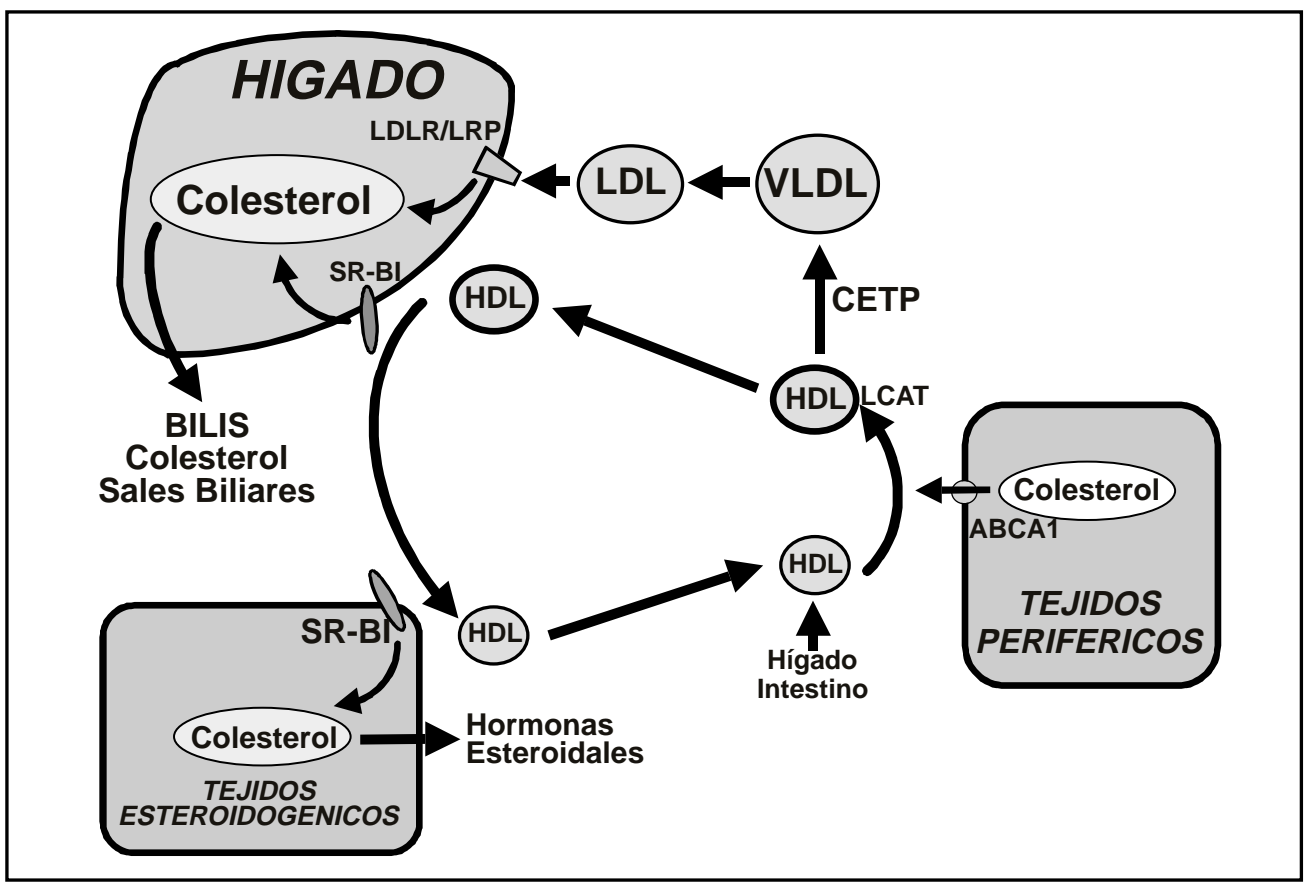

Figura 1. Metabolismo de HDL Las HDL nacientes sintetizadas en el hígado y en el intestino remueven eficientemente colesterol libre desde las células de los tejidos periféricos por mecanismos dependientes de la actividad del transportador ABCA1. Enseguida, las partículas de HDL son procesadas por la enzima LCAT para generar la formación de HDL enriquecidas en ésteres de colesterol. Estas partículas de HDL maduras tienen al menos tres destinos metabólicos: 1) la enzima CETP estimula la transferencia de ésteres de colesterol desde HDL a VLDL, las cuales se transforman en LDL y son catabolizadas por vía del receptor de LDL o el receptor LRP; 2) las partículas de HDL son fuente directa de ésteres de colesterol para la célula por medio de receptores endocíticos de la familia del receptor de LDL o receptores específicos de HDL todavía desconocidos (no mostrado); y 3) las HDL entregan colesterol directamente al hígado y los tejidos esteroidogénicos a través del proceso de captación selectiva de colesterol HDL mediado por el receptor SR-BI. El flujo del colesterol desde los tejidos periféricos por medio de las HDL plasmáticas hacia el hígado se conoce como transporte reverso de colesterol. Mientras el colesterol HDL removido por el hígado puede ser destinado para secreción biliar, el colesterol captado desde las HDL en los tejidos esteroidogénicos se utiliza para la síntesis de hormonas esteroidales ABCA1, ATP-binding cassette transporter class A type 1; CETP, cholesteryl ester transfer protein; HDL, lipoproteínas de alta densidad; LCAT, lecithin: cholesterol acyltransferase; LDL, lipoproteínas de baja densidad; LDLR, receptor de LDL; LRP, proteína relacionada al receptor LDL; SR-BI, scavenger receptor class B type I; VLDL, lipoproteínas de muy baja densidad. 
plasmática muy reducida de CETP ${ }^{11}$. El efecto de la actividad de la CETP sobre el desarrollo de aterosclerosis es todavía controvertido y dependería de la presencia de otros estados dislipidémicos asociados ${ }^{12}$.

Como segundo mecanismo catabólico, las HDL son fuente directa de colesterol para las células del organismo a través de la endocitosis y la degradación intracelular mediada por receptores de la superficie celular ${ }^{13}$. Las partículas de HDL de mayor tamaño y enriquecidas en colesterol adquieren apo E y serían endocitadas por los receptores de la familia del receptor de $\mathrm{LDL}^{14}$. Se postula que existe también un mecanismo endocítico degradativo de HDL, independiente de la apo E y del receptor de $\mathrm{LDL}^{15}$. La identidad de los genes y las proteínas respectivas involucradas en este proceso son temas de investigación activa.

El tercer mecanismo de catabolismo de las HDL es la captación celular selectiva del colesterol HDL, sin internalización ni degradación de la partícula lipoproteica ${ }^{13}$ (Figura 1). Este proceso metabólico explica el 20-30\% del clearance plasmático total del cHDL en animales que expresan CETP $^{16}$, sugiriendo que esta vía jugaría un importante papel en el metabolismo de HDL en humanos. Este proceso ocurre predominantemente en el hígado y es mediado por el receptor SR-BI (scavenger receptor class B type I) ${ }^{17}$. Varios estudios in vitro y en modelos animales han demostrado la importancia de SR-BI en el metabolismo de HDL y que la expresión de este receptor en el hígado controla los niveles plasmáticos del cHDL y su secreción hacia la vía biliari8-20. También, se ha establecido la relevancia de SR-BI en la patogenia de la ateroesclerosis en el ratón ${ }^{20}$. De hecho, la deficiencia simultánea de SR-BI y apo E en ratones, constituye uno de los pocos modelos animales de ateroesclerosis que desarrolla cardiopatía isquémica con trombosis coronaria, infartos miocárdicos múltiples, disfunción ventricular izquierda y muerte prematura ${ }^{21}$. Es muy probable que la expresión de SR-BI en humanos también juegue un papel importante en el metabolismo de HDL y la patogenia de la ateroesclerosis ${ }^{19,20}$.

El colesterol movilizado por las HDL desde los tejidos periféricos hacia el hígado por las vías metabólicas descritas, constituye el fenómeno denominado "transporte reverso de colesterol"22. El cHDL captado en el hígado puede ser utilizado para secreción biliar, tanto como colesterol libre 0 transformado en sales biliares. Este flujo de cHDL desde el plasma hacia la bilis constituye el principal mecanismo de remoción de colesterol del organismo ${ }^{22}$ y explicaría el papel clave que juegan las HDL en la regulación de la homeostasis del colesterol corporal.

\section{AlteRNATIVAS FARMACOLÓGICAS PARA EL MANEJO DEL METABOLISMO DE HDL \\ Y DEL RIESGO CARDIOVASCULAR ATEROESCLERÓTICO}

Dentro de las alternativas farmacológicas disponibles, los fibratos y la niacina pueden aumentar los niveles de cHDL hasta 30\%, las estatinas entre 5 y $10 \%$ y las resinas secuestrantes de sales biliares entre 3 y 5\%. El análisis post-hoc de varios estudios que usaron estas drogas hipolipemiantes, ha sugerido que parte del efecto beneficioso de estos fármacos sobre la enfermedad coronaria se podría explicar por el aumento observado en los niveles de cHDL, independiente de cambios en el cLDL y los triglicéridos ${ }^{23,24}$. Sin embargo, ninguno de estos protocolos tuvo como objetivo primario el manejo farmacológico del metabolismo de HDL para evaluar su impacto sobre los eventos cardiovasculares.

El reciente estudio VA-HIT (Veterans Administration HDL Intervention Trial ${ }^{25}$ tuvo como objetivo primario analizar el efecto del gemfibrozilo (1.200 mg/día) en pacientes con cHDL bajo $40 \mathrm{mg} /$ dL y enfermedad coronaria conocida, sobre la recurrencia de cardiopatía isquémica clínica durante un seguimento de aproximadamente 5 años. Comparado con el grupo placebo, el gemfibrozilo aumentó el cHDL en $6 \%$ y redujo los triglicéridos en 31\%, sin modificar los niveles de cLDL. Clínicamente, el gemfibrozilo disminuyó en $24 \%$ la incidencia combinada de infarto miocárdico no fatal, muerte por enfermedad coronaria y eventos cerebrovasculares isquémicos ${ }^{25}$ (Tabla 2). El análisis de regresión multivariada demostró que los niveles de cHDL alcanzados durante el tratamiento con gemfibrozilo, se correlacionaron en forma inversa con los eventos coronarios, independiente del cLDL y los triglicéridos ${ }^{25}$. Es importante destacar que un porcentaje importante de los pacientes con cHDL bajo eran obesos, diabéticos, hipertensos o presentaban resistencia insulínica ${ }^{26}$. Por el contrario, el 
Tabla 2. Prevención secundaria de eventos cardiovasculares isquémicos en pacientes con colesterol H D L bajo

\begin{tabular}{|lcccc|}
\hline Estudio clínico & Pacientes $(\mathrm{n})$ & Seguimiento & Redución del riesgo & $\mathrm{p}$ \\
\hline VA-HIT & 2.531 & 5,1 años & $-22 \%$ & 0,006 \\
$\begin{array}{l}(\approx 20 \% \text { con diabetes } \\
\text { o síndrome metabólico })\end{array}$ & & & \\
BIP (sin diabetes) & 3.090 & 6,2 años & $-7 \%$ & $n s$ \\
BIP subgrupo con & & & $-40 \%$ & 0,02 \\
$\quad$ triglicéridos $>200 \mathrm{mg} / \mathrm{dL}$ & & & & \\
\hline
\end{tabular}

n: número de pacientes. p: probabilidad estadísticas. ns: no significativo.

estudio BIP (Bezafibrate Infarction Prevention)27, donde pacientes con enfermedad coronaria establecida, cHDL $<45 \mathrm{mg} /$ dL y colesterol total moderadamente elevado (180-250 $\mathrm{mg} / \mathrm{dL})$ fueron randomizados para recibir placebo o bezafibrato (400 mg/día) por un promedio de 6,2 años, sólo mostró una tendencia no significativa a menor incidencia de nuevos eventos cardiovasculares en el grupo tratado con fibratos (Tabla 2). Sin embargo, un análisis post-hoc en el subgrupo de pacientes con triglicéridos basales $>200 \mathrm{mg} / \mathrm{dL}$, estableció que el bezafibrato disminuyó la probabilidad de nuevos eventos en aproximadamente $40 \%{ }^{27}$ (Tabla 2). Por lo tanto, los estudios VA-HIT y BIP indican que los fibratos serían particularmente útiles en los pacientes con cHDL bajo y en el contexto de una dislipidemia asociada al síndrome metabólico ${ }^{27}$.

Sin embargo, ninguno de estos estudios presentaba los niveles bajos de cHDL como única variable independiente y determinante del riesgo de patología cardiovascular. La falta de este tipo de estudios se debe a: 1) la dificultad existente para separar los niveles de cHDL de los de triglicéridos plasmáticos y otros índices metabólicos ya que estos parámetros están biológicamente relacionados, y 2) la ausencia de medidas no farmacológicas y farmacológicas eficaces para modificar selectiva y significativamente el metabolismo de las HDL Por lo tanto, los estudios de intervención sobre el cHDL no han permitido todavía establecer claramente si el efecto beneficioso de los fibratos sobre los eventos cardiovasculares se debe a los cambios en los niveles de cHDL versus los niveles de triglicéridos ( $p$ ej.: estudios VA-HIT o BIP) o la concomitancia de resistencia insulínica ( $p$ ej.: estudio VA-HIT).

Más recientemente, la infusión endovenosa semanal de la variante Milano de la apo A-I en pacientes con enfermedad coronaria establecida y evaluados con ultrasonografía intravascular a las 5 semanas, demostró una regresión significativa de la ateromatosis coronaria ${ }^{28}$. Este estudio constituye la primera prueba directa y específica del efecto protector cardiovascular de las HDL en humanos, atribuible probablemente a un aumento transitorio en los niveles de cHDL y en el transporte reverso de colesterol ${ }^{29,30}$. Además, este trabajo sugiere la posible utilidad de la infusión de HDL por corto plazo asociada a terapia hipolipemiante, para prevenir la recurrencia precoz de eventos coronarios isquémicos ${ }^{31}$. Actualmente, existen estudios que están evaluando la infusión de péptidos sintéticos de la apo A-I y la reinyección de HDL autóloga post-delipidación ex vivo.

Otra aproximación usada para inducir un aumento significativo y más sostenido de los niveles plasmáticos de cHDL, es la inhibición de la actividad de la enzima CETP. La inhibición farmacológica de CETP no sólo ha demostrado un aumento en el cHDL, sino que, además, una disminución de la ateromatosis en animales ${ }^{12}$. Un estudio inicial en humanos, usando $900 \mathrm{mg} /$ día de JTT-705, un inhibidor farmacológico de la CETP, por 4 semanas en sujetos sanos levemente hipercolesterolémicos demostró un aumento de $34 \%$ en el cHDL con una disminución de $7 \%$ en el $\mathrm{CLDL}^{32}$. En estudios más recientes, se ha evaluado el efecto de torcetrapib, otro inhibidor de la CETP, 
en sujetos con cHDL normal o bajo. El uso de torcetrapib (10-240 mg/día) disminuyó progresivamente la actividad de la CETP sin modificar los niveles de colesterol plasmático total, pero causando un aumento en los niveles de cHDL de 16 a $91 \%$ en sujetos con cHDL normal y de 46 a $106 \%$ en pacientes con cHDL bajo, reduciendo el cLDL en 8 a 42\%33,34. Nuevos estudios deberán confirmar el efecto de JTT-705 y torcetrapib sobre el fenotipo lipoproteico, optimizar las dosis para uso clínico sin producir efectos colaterales y establecer el beneficio clínico en prevención cardiovascular primaria o secundaria ${ }^{35}$. Además, el uso combinado de JTT-705 o torcetrapib con estatinas, podría constituir una nueva alternativa para mejorar la efectividad ya demostrada, aunque parcial, de estos últimos fármacos en el manejo del riesgo cardiovascular ateroesclerótico.

El análisis de la regulación de ABCA1, CETP y SR-BI ha permitido establecer que estas proteínas están bajo control de una serie de receptores nucleares, destacando los PPAR (peroxisome proliferator activated receptors) y LXR (liver X receptor alpha $)^{36}$. Así, los fibratos y las glitazonas son capaces de estimular la expresión de ABCA1 y el eflujo de colesterol desde los macrófagos hacia las $\mathrm{HDL}^{37}$. Más recientemente, nuevos fármacos que activan los factores de transcripción PPARß/ $\delta$ y LXR $\alpha$ también aumentan los niveles de ABCA1 en los macrófagos y los niveles plasmáticos de cHDL $^{38,39}$. Por otro lado, los agonistas de LXR $\alpha$ además estimulan la expresión de $\operatorname{CETP}^{40}$ y SR$\mathrm{BI}^{41}$, lo cual facilitaría el transporte reverso de colesterol tanto indirecto como directo y su excreción biliar en roedores ${ }^{42}$ (Figura 2). Varios de estos nuevos fármacos que facilitan el transporte de colesterol mediado por la HDL han demostrado también actividad anti-aterogénica en modelos animales $^{43}$. Esperamos que estas novedosas altemativas farmacológicas que permiten un manejo integral del metabolismo de HDL se puedan aplicar y demostrar su beneficio clínico en humanos.

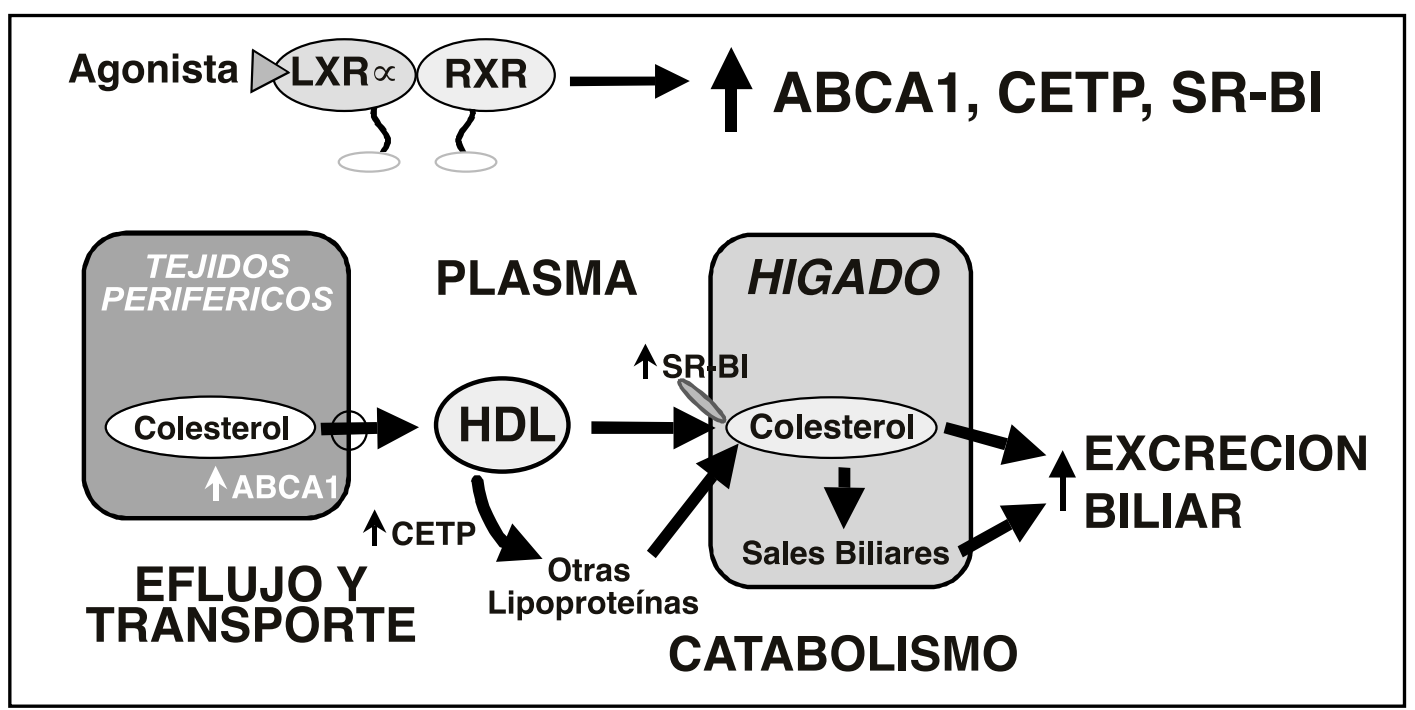

Figura 2. Efecto de la activación del receptor nuclear LXR $\alpha$ sobre el transporte reverso de colesterol. LXR $\alpha$ es un receptor nuclear heterodimérico que se activa por la unión de agonistas farmacológicos y determina un aumento en la expresión de varios genes en diferentes tejidos. La activación de LXR $\alpha$ aumentan los niveles de ABCA1 en los macrófagos y el eflujo de colesterol celular hacia las HDL. Los agonistas de LXR $\alpha$ también estimulan la expresión de CETP en el plasma y SR-BI en el hígado, todo lo cual facilitaría el transporte reverso tanto indirecto como directo de colesterol desde la periferia hacia el hígado. La estimulación de LXR $\alpha$ finalmente incrementa la síntesis de sales biliares y la excreción biliar de colesterol, lo cual lleva a una mayor eliminación de colesterol a través de la vía biliar. Por lo tanto, LXR $\alpha$ permite regular integralmente el metabolismo del colesterol HDL. 


\section{CONSIDERACIONES CLÍNICAS}

Aunque la evidencia epidemiológica sugería la importancia de las HDL en la patogenia de la ateroesclerosis, las primeras recomendaciones del National Cholesterol Education Program Adult Treatment Panel I (NCEP-ATPI) (1988), de Estados Unidos no consideró la medición rutinaria de los niveles de cHDL, su importancia como factor de riesgo de enfermedad coronaria, ni su utilización en las decisiones de manejo de los pacientes dislipidémicos ${ }^{44}$. El informe NCEP-ATPI fue criticado por esta omisión, lo que fue reconocido por el panel de expertos ${ }^{45}$ y llevó a que las nuevas recomendaciones de los NCEP-ATPII (1993) ${ }^{46}$ y NCEP-ATPIII (2001) ${ }^{47}$ incluyeran explícitamente medidas relacionadas con el cHDL De hecho, varias publicaciones adicionales han enfatizado la importancia de la pesquisa, los criterios de diagnóstico y el manejo de los pacientes con niveles bajos de $\mathrm{CHDL}^{23,48-55}$.

Actualmente, se recomienda la medición rutinaria de los niveles plasmáticos de cHDL en la evaluación del riesgo cardiovascular y se considera como nivel aceptable un colesterol-HDL $>40$ $\mathrm{mg} / \mathrm{dL}^{46}$. El nivel de cHDL $<40 \mathrm{mg} / \mathrm{dL}$ es un factor de riesgo mayor para enfermedad coronaria ${ }^{47}$. Por otro lado, los niveles de cHDL también forman parte de los criterios introducidos por el NCEPATPIII para calcular el riesgo cardiovascular de cada paciente ${ }^{47}$. Dado que un nivel de cHDL elevado generalmente protege contra la aterosclerosis, un cHDL $>60 \mathrm{mg} / \mathrm{dL}$ es considerado como un factor protector que permite contrarrestar la presencia de otro factor de riesgo ${ }^{46,47}$.

Las recomendaciones del NCEP-ATPIII no proponen ningún objetivo terapéutico específico para el cHDL y, aun en presencia de cHDL bajo, el tratamiento del cLDL constituye el objetivo terapéutico primario ${ }^{47}$. Si se tratan farmacológicamente pacientes dislipidémicos con $\mathrm{CHDL}<40 \mathrm{mg} / \mathrm{dL}$, el NCEP-ATPIII recomienda el uso de medicamentos que eleven los niveles de HDL, tales como los fibratos y el ácido nicotínico ${ }^{47}$. Todavía no se ha establecido el beneficio del tratamiento farmacológico del cHDL bajo aislado, en pacientes que no tienen otros trastornos lipídicos ni otros factores de riesgo ateroesclerótico. Sin embargo, siempre se deben recomendar en estos pacientes medidas generales (reducción de peso, suspensión del tabaquismo y ejercicio aeróbico) para cambiar el estilo de vida, ya que elevan los niveles de cHDL. Por otro lado, se deben evitar medicamentos que reducen el cHDL como betabloqueadores, progestágenos, corticoides y anabólicos.

El NCEP-ATPIII recomienda el tratamiento farmacológico del cHDL bajo aislado en casos de cardiopatía coronaria conocida o en pacientes con equivalentes de riesgo coronario ${ }^{47}$. Esta recomendación ha sido apoyada por el estudio VA-HIT donde se demostró que pacientes coronarios con cHDL bajo y niveles de cLDL aceptables asociados a diabetes 0 en el contexto de un síndrome metabólico se beneficiaron con el uso de gemfibrozilo ${ }^{25}$. Este estudio constituye la primera evidencia protocolizada prospectiva que apoya el tratamiento farmacológico de este grupo de pacientes que constituyen un porcentaje significativo de la población coronaria. Esta recomendación también se apoya en los análisis post-hoc del Helsinki Heart Study y del estudio BIP23,27.

Estas pautas generales han sido recientemente reforzadas por las recomendaciones de un grupo de expertos internacionales sobre la importancia del cHDL en la prevención y el tratamiento de la enfermedad coronaria isquémica ${ }^{23}$. Este grupo ha propuesto: 1) obtener niveles de cHDL $>40 \mathrm{mg} / \mathrm{dL}$ en los pacientes con enfermedad cardiovascular isquémica establecida o en aquéllos sin enfermedad, pero con alto riesgo cardiovascular determinado por la presencia de diabetes mellitus o síndrome metabólico, 2) aplicar las medidas generales ya mencionadas más una dieta con aporte moderado de grasa insaturada, 3) considerar el uso de fibratos o niacina en pacientes coronarios con cHDL bajo aislado que no requieren estatinas o que ya están siendo tratados con ellas de acuerdo a las recomendaciones establecidas para el manejo del cLDL.

Por lo tanto, actualmente disponemos de evidencia que apoya el beneficio del tratamiento farmacológico de ciertos subgrupos de pacientes con cHDL bajo. Esperamos que nuevos estudios con las drogas actualmente disponibles o con nuevos fármacos permitan ampliar estas recomendaciones del manejo de pacientes con bajos niveles de cHDL disminuyendo su riesgo cardiovascular a corto y largo plazo. 


\section{CONCLUSIONES}

Los importantes avances logrados recientemente en la comprensión del metabolismo del cHDL y su regulación, junto con el desarrollo de nuevos fármacos que manipulan específicamente los niveles de cHDL y los resultados de los primeros

\section{REFERENCIAS}

1. Mascie-Taylor CG, Karim E. The burden of chronic disease. Science 2003; 302: 1921-2.

2. Thompson GR, Barter PJ. Clinical lipidology at the end of the millennium. Curr Opin Lipidol 1999; 10: 521-6.

3. BARTER PJ, Rye KA. High density lipoproteins and coronary heart disease. Atherosclerosis 1996; 121: 1-12.

4. GoRdon DJ, RifKInd BM. High-density lipoprotein, the clinical implications of recent studies. N Engl J Med 1989; 321: 1311-6.

5. SCHAEFER EJ. Familial lipoprotein disorders and premature coronary artery disease. Med Clin North Am 1994; 78: 21-39.

6. Kwiterovich PO Jr. The antiatherogenic role of high-density lipoprotein cholesterol. Am J Cardiol 1998; 82: 13Q-21Q.

7. BREwer HB JR, Santamarina-Fojo S. Clinical significance of high-density lipoproteins and the development of atherosclerosis: focus on the role of the adenosine triphosphate-binding cassette protein A1 transporter. Am J Cardiol 2003; 92: 10K-16K.

8. Joyce C, Freeman L, Brewer HB Jr, Santamarina-Fojo S. Study of ABCA1 function in transgenic mice. Arterioscler Thromb Vasc Biol 2003; 23: 965-71.

9. Peelman F, Vandekerckhove J, Rosseneu M. Structure and function of lecithin cholesterol acyltransferase: new insights from structural predictions and animal models. Curr Opin Lipidol 2000; 11: 155-60.

10. TALL AR, Jiang X, Luo Y, Silver D. Lipid transfer proteins, HDL metabolism, and atherogenesis. Arterioscler Thromb Vasc Biol 2000; 20: 1185-8.

11. Cuevas A, Alvarez V, Acosta AM, Altayo M, MonteRo J, Rigotтi A. Mecanismos de hipo e hiperalfalipoproteinemia en individuos adultos chilenos. Rev Méd Chile 2004; 132: 421-8. estudios clínicos enfocados hacia el manejo primario del cHDL permiten vislumbrar la aplicación de nuevas estrategias terapéuticas para las dislipidemias. Estos nuevos tratamientos podrían tener un impacto adicional al beneficio ya demostrado de las estatinas sobre la enfermedad cardiovascular ateroesclerótica.

12. Barter PJ, Brewer HB Jr, Chapman MJ, Hennekens CH, RADER DJ, TALL AR. Cholesteryl ester transfer protein: a novel target for raising HDL and inhibiting atherosclerosis. Arterioscler Thromb Vasc Biol 2003; 23: 160-7.

13. Pittman RC, Steinberg D. Sites and mechanisms of uptake and degradation of high density and low density lipoproteins. J Lipid Res 1984; 25: 1577-785.

14. LEBLOND L, MARCEL YL Uptake of high density lipoprotein cholesterol ester by HepG2 cells involves apolipoprotein E localized on the cell surface. J Biol Chem 1993; 268: 1670-6.

15. García A, Barbaras $R$, Colet $X$, Bogyo A, Chap $H$, PERRET B. High density lipoprotein 3 receptordependent endocytosis pathway in a human hepatoma cell line (HepG2). Biochemistry 1996; 35: 13064-71.

16. Goldberg DI, Beltz WF, Pittman RC. Evaluation of pathways for the cellular uptake of high density lipoprotein cholesterol esters in rabbits. J Clin Invest 1991; 87: 331-46.

17. Acton S, Rugotti A, Landschulz KT, Xu S, Hobbs $\mathrm{HH}, \mathrm{KRIEGER} \mathrm{M}$. Identification of scavenger receptor SR-BI as a high density lipoprotein receptor. Science 1996; 271: 518-20.

18. Rigotti A, Krieger M. Getting a handle on "good" cholesterol with the high-density lipoprotein receptor. N Engl J Med 1999; 341: 2011-3.

19. Rigotti A, Miettinen HE, Krieger M. The role of the high-density lipoprotein receptor SR-BI in the lipid metabolism of endocrine and other tissues. Endocr Rev 2003; 24: 357-87.

20. Trigatti BL, Krieger M, Rigotti A. Influence of the HDL receptor SR-BI on lipoprotein metabolism and atherosclerosis. Arterioscler Thromb Vasc Biol 2003; 23: 1732-8.

21. Braun A, Trigatti BL, Post MJ, Sato K, Simons M, EDELBERG JM ET AL. Loss of SR-BI expression leads 
to the early onset of occlusive atherosclerotic coronary artery disease, spontaneous myocardial infarctions, severe cardiac dysfunction, and premature death in apolipoprotein E-deficient mice. Circ Res 2002; 90: 270-6.

22. RADER DJ. Regulation of reverse cholesterol transport and clinical implications. Am J Cardiol 2003; 92: 42J-49J.

23. Sacks FM; EXPert Group on HDL Cholesterol. The role of high density lipoprotein (HDL) cholesterol in the prevention and treatment of coronary heart disease: expert group recommendations. Am J Cardiol 2002; 90: 139-43.

24. SACKS FM. The relative role of low density lipoprotein cholesterol and high density lipoprotein cholesterol in coronary artery disease: evidence from large scale statin and fibrate trials. Am J Cardiol 2001; 88: 14N-18N.

25. Rubins HB, Robins SJ, Cowns D, Fye CL, Anderson JW, Elam MB Eт Al. Gemfibrozil for the secondary prevention of coronary heart disease in men with low levels of high density lipoprotein cholesterol. N Eng J Med 1999; 341: 410-8.

26. Rubins HB, Robins SJ. Conclusions from the VAHIT study. Am J Cardiol 2000; 86: 543-4.

27. The BIP (Benzafibrato Infarction Prevention) Study GRouP. Secondary Prevention by Raising HDL Cholesterol and Reducing Triglycerides in Patients With Coronary Heart Disease. Circulation 2000; 102: 21-7.

28. Nissen SE, Tsunoda T, Tuzcu EM, Schoenhagen P, COOPER CJ, YAsin M ET AL. Effect of recombinant Apo A-I Milano on coronary atherosclerosis in patients with acute coronary syndromes: a randomized controlled trial. JAMA 2003; 290: 2292-300.

29. Eriksson M, Carlson LA, Miettinen TA, Angelin B. Stimulation of fecal steroid excretion after infusion of recombinant proapolipoprotein A-I. Potential reverse cholesterol transport in humans. Circulation 1999; 100: 594-8.

30. Nanjee MN, Cooke CJ, Garvin R, Semeria F, Lewis G, OLSZEWSKI WL ET AL. Intravenous apo A-I/lecithin discs increase pre beta HDL concentration in tissue fluid and stimulate reverse cholesterol transport in humans. J Lipid Res 2001; 42: 158693.

31. RADER DJ. High density lipoproteins as an emerging therapeutic target for atherosclerosis. JAMA 2003; 290: 2322-4.
32. de Grooth GJ, Kuivenhoven JA, Stalenhoef AF, de GraAF J, Zwinderman AH, Posma JL et al. Efficacy and safety of a novel cholesteryl ester transfer protein inhibitor, JTT-705, in humans: a randomized phase II dose-response study. Circulation 2002; 105: 2159-65.

33. Ciark RW, Sutfin TA, Ruggeri RB, Wilauer AT, Sugarman ED, Magnus-Ary ITey G et aL. Raising high-density lipoprotein in humans through inhibition of cholesteryl ester transfer protein: an initial multidose study of torcetrapib. Arterioscler Thromb Vasc Biol 2004; 24: 490-7.

34. Brousseau ME, Schaefer EJ, Wolfe ML, Bloedon LT, Digenio AG, Ciark RW et al. Effects of an inhibitor of cholesteryl ester transfer protein on HDL cholesterol. N Engl J Med 2004; 350: 1505-15.

35. BREWER HB JR. Increasing HDL Cholesterol Levels. N Engl J Med 2004; 350: 1491-4.

36. Rigotтi A. Los receptores nucleares como nuevas dianas terapéuticas para un manejo integral de las dislipidemias. Clin Invest Arterioscl 2003; 15: 156-63.

37. LAZAR MA. Progress in cardiovascular biology: PPAR for the course. Nat Med 2001; 7: 23-4.

38. Repa JJ, Turley SD, Lobaccaro JMA, Medina J, Li L, Lustig $\mathrm{K}$ et al. Regulation of absorption and ABC1 mediated efflux of cholesterol by RXR heterodimers. Science 2000; 289: 1524-9.

39. Oliver W, Shenk J, Snaith, Russell C, Plunket K, BodKIN N ET AL. A selective peroxisome proliferator-activated receptor $\delta$ agonist promotes reverse cholesterol transport. Proc Natl Acad Sci USA 2001; 98: 5306-11.

40. LuO Y, TALL AR. Sterol upregulation of human CETP expression in vitro and in transgenic mice by an LXR element. J Clin Invest 2000; 105: 51320.

41. Malerod L, Juvet LK, Hanssen-Bauer A, Eskild W, BERG T. Oxysterol-activated LXRalpha/RXR induces hSR-BI-promoter activity in hepatoma cells and preadipocytes. Biochem Biophys Res Commun 2002; 299: 916-23.

42. Milatt LJ, Bocher V, Fruchart JC, Staels B. Liver X receptors and the control of cholesterol homeostasis: potential therapeutic targets for the treatment of atherosclerosis. Biochim Biophys Acta 2003; 1631: 107-18.

43. Ricote M, VaLedor AF, Glass CK. Decoding transcriptional programs regulated by PPARs and 
LXRs in the macrophage: effects on lipid homeostasis, inflammation, and atherosclerosis. Arterioscler Thromb Vasc Biol 2004; 24: 230-9.

44. Report of the National Cholesterol Education Program Expert Panel on Detection, Evaluation, and Treatment of High Blood Cholesterol in Adults. The Expert Panel. Arch Intern Med 1988; 148: 36-69.

45. Grundy SM, Goodman DW, Rifkind BM, Cieeman JI. The place of HDL in cholesterol management. A perspective from the National Cholesterol Educational Program. Arch Intern Med 1989; 149: 505-10.

46. Summary of the second report of the National Cholesterol Education Program (NCEP) Expert Panel on Detection, Evaluation, and Treatment of High Blood Cholesterol in Adults (Adult Treatment Panel II). JAMA 1993; 269: 3015-23.

47. Expert Panel on Detection, Evaluation, and Treatment of High Blood Cholesterol in Adults. Executive Summary of the Third Report of the National Cholesterol Education Program (NCEP) Expert Panel on Detection, Evaluation, And Treatment of High Blood Cholesterol In Adults (Adult Treatment Panel III). JAMA 2001; 285: 2486-97.

48. Rosenson RS. Low levels of high-density lipoprotein cholesterol (hypoalphalipoproteinemia). An approach to management. Arch Intern Med 1993; 153: 1528-38.

49. VeGA GL, GRUNDY SM. Hypoalphalipoproteinemia (low high density lipoprotein) as a risk factor for coronary heart disease. Curr Opin Lipidol 1996; 7: 209-16.

50. HaRPER CR, JaCobson TA. New perspectives on the management of low levels of high-density lipoprotein cholesterol. Arch Intern Med 1999; 159: 1049-1045

51. RADER DJ. Pathophysiology and management of low high-density lipoprotein cholesterol. Am J Cardiol 1999; 83: 22F-24F.

52. RADER DJ, Rosas S. Management of selected lipid abnormalities. Hypertriglyceridemia, low HDL cholesterol, lipoprotein(a), in thyroid and renal diseases, and post-transplantation. Med Clin North Am 2000; 84: 43-6.

53. Civeira F, Gonzalvo C. Tratamiento del colesterol HDL bajo. Clin Invest Arterioscl 2000; 12: 209-11.

54. WATTS GF. Treating patients with low high density lipoprotein cholesterol: choices, issues and opportunities. Curr Control Trials Cardiovasc Med 2001; 2: 118-22.

55. Тотн PP. High density lipoprotein and cardiovascular risk. Circulation 2004; 109: 1809-12.

Agradecimientos

Nuestro trabajo de investigación ha sido financiado por FONDECYT (Proyectos \#8990006 y \#1030416) y por el Proyecto SAVAL de Investigación Clínica de la Sociedad Médica de Santiago. 